\title{
Effect of implantation on engineered skeletal muscle constructs
}

\author{
Michael L. Williams ${ }^{1}$, Tatiana Y. Kostrominova ${ }^{2,5}$, Ellen M. Arruda ${ }^{1,3,4}$ and Lisa M. Larkin ${ }^{1,2 *}$ \\ ${ }^{1}$ Biomedical Engineering, University of Michigan, Ann Arbor, MI, USA \\ ${ }^{2}$ Molecular and Integrative Physiology, University of Michigan, Ann Arbor, MI, USA \\ ${ }^{3}$ Mechanical Engineering, University of Michigan, Ann Arbor, MI, USA \\ ${ }^{4}$ Program in Macromolecular Science and Engineering, University of Michigan, Ann Arbor, MI, USA \\ ${ }^{5}$ Department of Anatomy and Cell Biology, Indiana University School of Medicine-Northwest, Gary, IN, USA
}

\begin{abstract}
The development of engineered skeletal muscle would provide a viable tissue for replacement and repair of muscle damaged by disease or injury. Our current tissue-engineering methods result in three-dimensional (3D) muscle constructs that generate tension but do not advance phenotypically beyond neonatal characteristics. To develop to an adult phenotype, innervation and vascularization of the construct must occur. In this study, 3D muscle constructs were implanted into the hindlimb of a rat, along the sciatic nerve, with the sural nerve isolated, transected and sutured to the construct to encourage innervation. Aortic ring anchors were sutured to the tendons of the biceps femoris muscle so that the construct would move dynamically with the endogenous muscle. After 1 week in vivo, the constructs were explanted, evaluated for force production and stained for muscle, nerve and collagen markers. Implanted muscle constructs showed a developing capillary system, an epimysium-like outer layer of connective tissue and an increase in myofibre content. The beginning of $\alpha$-bungarotoxin clustering suggests that neuromuscular junctions (NMJs) could form on the implanted muscle, given more time in vivo. Additionally, the constructs increased maximum isometric force from $192 \pm 41 \mu \mathrm{N}$ to $549 \pm 103 \mu \mathrm{N}$ (245\% increase) compared to in vitro controls, which increased from $276 \pm 23 \mu \mathrm{N}$ to $329 \pm 27 \mu \mathrm{N}$ (25\% increase). These findings suggest that engineered muscle tissue survives 1 week of implantation and begins to develop the necessary interfaces needed to advance the phenotype toward adult muscle. However, in terms of force production, the muscle constructs need longer implantation times to fully develop an adult phenotype. Copyright (c) 2012 John Wiley \& Sons, Ltd.
\end{abstract}

Received 11 April 2011; Revised 12 September 2011; Accepted 3 November 2011

Keywords tissue engineering; implantation; skeletal muscle; innervation; vascularization

\section{Introduction}

The development of engineered skeletal muscle would provide a viable option for replacement and repair of damaged skeletal muscle in humans as well as a viable model for studying the effects of stressors and chemicals on muscle (Khodabukus et al., 2007). The current method for skeletal muscle repair, the muscle flap, is hindered by

*Correspondence to: L. Larkin, Molecular and Integrative Physiology, Biomedical Engineering, University of Michigan, Biomedical Science Research Building (BSRB), 109 Zina Pitcher Place, Room 2025, Ann Arbor, MI 48109-2200, USA. E-mail: llarkin@ umich.edu limited tissue availability and donor site morbidity. Our laboratory has developed a reproducible method for the development of scaffold-less engineered muscle constructs (Larkin et al., 2006). The engineered muscle we fabricate consists of a 3D construct with a fibroblastproduced extracellular matrix (ECM) and myotubes aligned along the axis of strain. This in vitro construct can generate force either spontaneously or in response to electrical field stimulation. Engineered skeletal muscle is limited in vitro in that it does not develop beyond a neonatal phenotype in terms of strength characteristics and tissue organization (Larkin et al., 2006; Huang et al., 2010).

Two interfaces that we aimed to establish in our implanted constructs are neural and vascular. Our laboratory has 
enhanced engineered muscle in vitro through the co-culture of muscle and fetal nerve cells (Larkin et al., 2005). Other work has been done to successfully enhance scaffolded engineered muscle function by mimicking neural stimulation through bioreactors that deliver cyclic electric pulses (Donnelly et al., 2010). In order for engineered muscles to grow to physiological sizes, a vascular network must be established. Dennis and Kosnik (2000) showed that myoblasts are unable to differentiate and proliferate more than $150 \mu \mathrm{m}$ away from oxygen and nutrient supplies. Other research groups have shown that the foreign body response observed during in vivo implantation supports the ingrowth of a capillary network into the implanted constructs (Beier et al., 2006; Saxena et al., 2001).

In addition to the benefits of neural and vascular interfaces, we expected our constructs to demonstrate a positive response to the cyclic mechanical stretching provided by the movement of the host muscles. Mechanical stimulation in vitro has been performed using bioreactors on scaffolded muscle tissue (Gonen-Wadmany et al., 2004; Powell et al., 2002) to enhance skeletal muscle phenotype. Gonen-Wadmany et al. (2004) showed that mechanical stimulation improves cell-mediated collagen compaction, resulting in improved cellular alignment. Thus, we hypothesized that the implantation of our muscle construct in an anatomical location to provide mechanical stretch during ambulation of the animal would enhance the phenotype when compared with the maintained in vitro construct.

The purpose of this research was to investigate the effect of 1 week of in vivo implantation on scaffold-less engineered skeletal muscle constructs with respect to contractile properties, structure, vascularization and innervation. We hypothesized that the 1 week of implantation of muscle constructs would shift the phenotype from neonatal toward adult and that muscle constructs would exhibit an increase in force production.

\section{Materials and methods}

\subsection{Animal model and animal care}

Tissue-engineering studies were carried out using muscle tissue from female Fischer 344 rats, obtained from Charles River Laboratories Inc. (Wilmington, MA, USA). All animals were acclimated to our colony conditions, i.e. light cycle and temperature, for 1 week prior to any procedure. The animals were fed Purina Rodent Chow 5001 laboratory chow and water ad libitum. Surgical procedures were performed to remove both soleus muscles and aorta from a subset of the female Fisher rats. These rats were obtained as retired breeders with an estimated age of 8-9 months. These harvested tissues were then used as an allogenic cell source for muscle precursor cells and anchoring tissues (aortic rings) for the constructs fabricated and implanted into the host animals. All surgical procedures were performed in an aseptic environment, with animals in a deep plane of anaesthesia induced by intraperitoneal (i.p.) injections of sodium pentobarbital $(65 \mathrm{mg} / \mathrm{kg})$. Supplemental doses of pentobarbital were administered as required to maintain an adequate depth of anaesthesia. All animal care and animal surgery procedures were in accordance with The Guide for Care and Use of Laboratory Animals (National Research Council, 1996); the experimental protocol was approved by the University Committee for the Use and Care of Animals.

\subsection{Preparation of media}

Unless otherwise indicated, all solutions and media were prepared and stored at $4^{\circ} \mathrm{C}$ prior to isolation and culture of cells and warmed to $37^{\circ} \mathrm{C}$ in a heated water bath immediately prior to use. Muscle growth medium (MGM) contained $300 \mathrm{mlF}-12$ Kaighn's Modification Nutrient Mixture (Gibco BRL, cat. no. 21127-022), $125 \mathrm{ml}$ Dulbecco's modified Eagle's medium (DMEM; Gibco BRL, cat. no. 11995-065), $75 \mathrm{ml}$ fetal bovine serum (FBS; Gibco BRL, cat. no. 10437-028), $2.4 \mathrm{ng} / \mathrm{ml}$ basic fibroblast growth factor (bFGF; Peprotech) and $5 \mathrm{ml}$ antibiotic-antimycotic (ABAM; Gibco BRL, cat. no. 15240-062). Muscle differentiation medium (MDM) was composed of $330 \mathrm{ml}$ M199 (Gibco BRL, cat. no. 11150-059), $140 \mathrm{ml}$ DMEM, $33 \mathrm{ml}$ FBS, $500 \mu \mathrm{l}$ insulin transferring selenite-X (ITS-X; 1/1000 dilution), $362 \mu \mathrm{l}$ $50 \mu \mathrm{M}$ ascorbic acid 2-phosphate (Sigma-Aldrich), and $5 \mathrm{ml}$ ABAM (Gibco BRL, cat. no. 15240-062). Transport medium (TM) consisting of Dulbecco's phosphate-buffered saline (DPBS), pH 7.2 (Gibco BRL, cat. no. 14190-144), supplemented with $2 \%$ ABAM, was used to transfer freshly isolated tissues from the surgical suite to the tissue culture facilities for isolation.

\subsection{Preparation of construct dishes}

Muscle constructs were engineered in individual $60 \mathrm{~mm}$ plates, as described previously (Larkin et al., 2006). Each $60 \mathrm{~mm}$ plate was coated with $5 \mathrm{ml}$ Sylgard (Dow Chemical Corp., Midland, MI, USA; type 184 silicon elastomer) and allowed to cure for 3 weeks prior to use; $1-7$ days prior to use, Sylgard-coated plates were coated with laminin at $0-0.71 \mu \mathrm{g} / \mathrm{cm}^{2} /$ plate [20 $\mu \mathrm{g}$ Natural Mouse Laminin (Gibco BRL, cat. no. 23017-015) and $4 \mathrm{ml}$ Dulbecco's phosphatebuffered saline (DPBS), pH 7.2 (Gibco BRL, cat. no. 14190-144) per plate], and left to dry for $48 \mathrm{~h}$. Salt crystals were dissolved and removed by rinsing the plates with $4 \mathrm{ml}$ DPBS. The plates were then filled with $3 \mathrm{ml}$ previously described MGM and decontaminated with UV light (wavelength $253.7 \mathrm{~nm}$ ) for $60 \mathrm{~min}$ and placed in a $37^{\circ} \mathrm{C} 5 \% \mathrm{CO}_{2}$ incubator for up to 1 week prior to plating the muscle cells.

\subsection{Preparation of acellular aorta ring anchors}

The descending aortas of Fischer 344 rats were surgically removed under aseptic conditions. Cells were removed from the aorta, leaving just the ECM, by immersing the 
tissue sequentially in one of five detergent solutions over a period of 15 days, as outlined in Haase et al. (2003). The compositions of the solutions were as follows: solution 1 , $7.3 \mathrm{~g}$ ethylenediamine tetra-acetic acid (EDTA), $0.5 \mathrm{~g}$ sodium azide $\left(\mathrm{NaN}_{3}\right), 800 \mathrm{ml}$ glycerol, $200 \mathrm{ml} \mathrm{0.9 \%} \mathrm{NaCl}$; solution 2, $25 \mathrm{~g}$ sodium deoxycholate; $0.26 \mathrm{~g} \mathrm{NaN}_{3}, 600 \mathrm{ml}$ distilled and deionized water; solution 3, $10 \mathrm{~g}$ sodium dodecylsulphate (SDS), $0.52 \mathrm{~g} \mathrm{NaN}_{3}$ and $1000 \mathrm{ml}$ distilled and deionized water; solution 4, $0.5 \mathrm{~g} \mathrm{NaN}_{3}, 1000 \mathrm{ml} \mathrm{0.9 \%}$ saline; solution 5, $15 \mathrm{ml}$ Triton $\mathrm{X}-100,0.25 \mathrm{~g} \mathrm{NaN}_{3}$ and $485 \mathrm{ml}$ distilled water.

\subsection{Dissection of muscle and isolation of muscle cells}

Both soleus muscles were surgically removed under aseptic conditions, weighed and sterilized in 70\% ETOH. Prior to dissociation, muscles were incubated in TM (at a concentration of $5 \mathrm{ml} / \mathrm{muscle}$ dissected) for $5 \mathrm{~min}$. A $20 \mathrm{ml}$ dissociation solution consisting of $32 \mathrm{U}$ dispase (Sigma, cat. no. P-3417; 0.4 U/mg) and 2390 U type 4 collagenase (Gibco BRL, cat. no. 17104-019), 239 U/mg)/ml Ham's F12 (Gibco BRL, cat. no. 11765-054) was utilized. Two soleus muscles were dissociated for each $20 \mathrm{ml}$ solution for a minimum of a $1.5 \mathrm{~h}$ time period. The dissociated cell solution was poured through a $100 \mu \mathrm{m}$ filter, centrifuged and aspirated prior to being resuspended in $15 \mathrm{ml}$ MGM and preplated overnight in a $100 \mathrm{~mm}$ tissue culture dish. The supernatant from the preplate was removed and brought to a density of $1.3 \times 10^{5}$ cells $/ \mathrm{ml} ; 3 \mathrm{ml}$ of the cell suspension were plated in each of the previously prepared laminin-coated culture dishes and placed in a $37^{\circ} \mathrm{C}, 5 \%$ $\mathrm{CO}_{2}$ incubator. The culture plates were not disturbed for at least $72 \mathrm{~h}$ to allow cell adherence to the plates.

\subsection{Preparation of muscle constructs}

Muscle constructs were fabricated as previously described (Larkin et al., 2006) with the exception that aortic rings were used as anchors instead of adult rat tail tendon. Briefly, 5 days after initial plating, the cells were fed with MGM every $48 \mathrm{~h}$ until the cells became confluent (approximately 7 days). Once the cells achieved confluence, they were fed with MDM every $48 \mathrm{~h}$ until the myocytes fused to form multinucleated myotubes that began to contract spontaneously. At this time point, the aortic rings were pinned as anchors with minutien pins onto the muscle monolayer at a separation distance of $15 \mathrm{~mm}$. The aortic rings acted as anchors and enabled the muscle monolayer to roll up into a 3D cylinder. Approximately 7 days later, a cylindrical 3D muscle construct was formed in each dish. The cells were fed MDM every $48 \mathrm{~h}$ during the co-culture of the muscle constructs. Five days after construct formation, the muscle constructs were tested for contractile function as described in Section 2.8 below. At the conclusion of functional tests, each specimen was snap-frozen in Tissue Freezing Media (Triangle Biological Sciences,
Durham, NC, USA) in isopentane chilled with dry ice, and stored at $-80^{\circ} \mathrm{C}$ for subsequent histology.

\subsection{Implantation of muscle constructs}

F344 retired breeder rats were anaesthetized using isoflourane; the analgesic, Carprofen $(5 \mathrm{mg} / \mathrm{kg}$ ) was administered following induction of anaesthesia and every $12 \mathrm{~h}$ for $48 \mathrm{~h}$ post-surgery. The left leg vastus lateralis and biceps femoris muscles were separated, exposing the sciatic nerve. Approximately $10 \mathrm{~mm}$ of sural nerve was separated from the peroneal and tibial nerves and from the surrounding connective tissue. A small surface blood vessel from the medial edge of the biceps was dissected, exposing a $10 \mathrm{~mm}$ section, with the proximal end open. Muscle constructs were surgically placed in the hindlimb alongside the femur. The transected ends of the sural nerve and blood vessel were placed next to the muscle belly and sutured to the construct to encourage innervation and vascularization of the construct. The aortic rings of the constructs were sutured with 9-0 suture silk to the proximal and distal tendons of the biceps femoris muscle, so that they would experience mechanical stresses and move dynamically with the endogenous muscle. The vastus lateralis and biceps femoris muscles were sutured together, using 7-0 suture (Ethicon), and the skin incision was closed using $9 \mathrm{~mm}$ autoclips (Becton-Dickinson). The animals were allowed to recover for 7 days. At the end of the 7 day implantation, the muscle constructs were dissected, placed in MDM and their contractile properties were measured.

\subsection{Contractile property testing}

Contractile properties were measured prior to (day 0) and 7 days after implantation of the muscle constructs. The protocol for measuring contractility of engineered muscle constructs was adapted from Dennis and colleagues (Dennis and Kosnik, 2000; Dennis et al., 2001; Kosnik et al., 2001) and Irintchev et al. (1998). Briefly, the pin on one end of the construct was freed from the Sylgard and attached to a force transducer with canning wax. For field stimulation of the entire construct, platinum wire electrodes were positioned on either side of the construct. The temperature of the construct was maintained at $37 \pm$ $1^{\circ} \mathrm{C}$, using a heated aluminium platform. The diameter of the construct was determined and used to calculate cross-sectional area, assuming a circular cross-section. Passive baseline force was measured as the average baseline passive force preceding the onset of stimulation. Twitches were elicited using a single $2.5 \mathrm{~ms}$ pulse at 10 , 30,60 and $90 \mathrm{~mA}$, whereas maximum tetanic force was determined using a $1 \mathrm{~s}$ train of $2.5 \mathrm{~ms}$ pulses at $90 \mathrm{~mA}$ and 10, 20, 40, 60 and $80 \mathrm{~Hz}$. Data files for each peak twitch force and peak tetanic force trace were recorded and subsequently analysed, using LabVIEW data acquisition software. Peak tetanic force was normalized for 
cross-sectional area to determine maximum specific force. Following the direct field stimulation of the entire construct, a micro-electrode was used to electrically stimulate the neural extensions projecting from the explanted constructs, using the same stimulation parameters as described above for the field stimulation.

\subsection{Histochemical and immunohistochemical analysis of $3 \mathrm{D}$ muscle constructs}

Unfixed muscle constructs were placed into TBS medium (Triangle Biological Sciences, Durham, NC, USA), frozen in cold isopentane and stored at $-80^{\circ} \mathrm{C}$ until needed. Samples were sliced in the muscle portion of the construct to obtain cross-sections with a cryostat at a thickness of approximately $12 \mu \mathrm{m}$, adhered to Superfrost Plus microscopy slides and used for staining. Sections were stained for general morphology observations with haematoxylin and eosin (H\&E), as previously described (Luna, 1968). For immunohistochemical analysis, frozen sections were fixed with ice-cold methanol for $10 \mathrm{~min}$ and rinsed with phosphate-buffered saline (PBS). Sections were blocked for 30 min with PBS-0.05\% Tween20 (PBST) containing 20\% calf serum (PBST-S) at room temperature. The sections were incubated overnight at $4^{\circ} \mathrm{C}$ with the primary antibodies diluted in PBST-S. Immunofluorescent staining with specific antibodies was performed to detect the presence of myosin heavy chains (MCH; MF-20 mouse monoclonal antibody 1:5 dilution, obtained from the Developmental Studies Hybridoma Bank, Iowa City, IA, USA), $\alpha$-actinin (mouse monoclonal antibody; 1:200 dilution; Sigma, St. Louis, MO, USA), nebulin (mouse monoclonal antibody, 1:100 dilution; Abcam, Cambridge, MA, USA), PAX7 (rabbit polyclonal antibody, 1:100 dilution; Abcam), collagen type 1 (rabbit polyclonal antibody, $5 \mu \mathrm{g} / \mathrm{ml}$; Chemicon International, Temecula, CA, USA), blood vessels (rabbit polyclonal anti-CD31 antibody, $10 \mu \mathrm{g} / \mathrm{ml}$; Abbiotec, San Diego, CA, USA), nerve fibres (rabbit polyclonal anti-S-100 antibody, 1:100 dilution; Abcam) and mouse monoclonal anti-pan-axonal neurofilament marker SMI312 antibody (1:500; Covance, Emeryville, CA, USA). Following three washes in PBST, a $1 \mathrm{~h}$ room temperature incubation with Cy3-conjugated anti-mouse or anti-rabbit antibody (Jackson ImmunoResearch Laboratory, West Grove, PA) was used for visualization. Following three washes in PBST, nuclei were stained by 5 min incubation with DAPI solution (Sigma) in PBST. According to previously described methods (Kostrominova, 2011), staining of sections with fluorescein-labelled wheat germ agglutinin (WGA, $5 \mu \mathrm{g} / \mathrm{ml}$ for $5 \mathrm{~min}$; Molecular Probes, Eugene, OR, USA) was used for visualization of connective tissue. For visualization of developing neuromuscular junctions, muscle sections were incubated for $10 \mathrm{~min}$ at room temperature in the solution of $\alpha$-bungarotoxin ( $\alpha$-BTX-rhodamine, $1 \mu \mathrm{g} / \mathrm{ml}$; Sigma). The sections were examined and photographed with a Leica microscope and cross-sections of the constructs were analysed using a software package, Image J.

\subsection{Statistics}

Values are presented as mean \pm SE. Statistical analysis was performed using Staveiw (SAS Institute Inc., Cary, NC, USA). A one-way analysis of variance (ANOVA) was conducted to compare the differences between muscle constructs implanted in vivo for 1 week and in vitro constructs. Differences were considered significant at $p<0.05$.
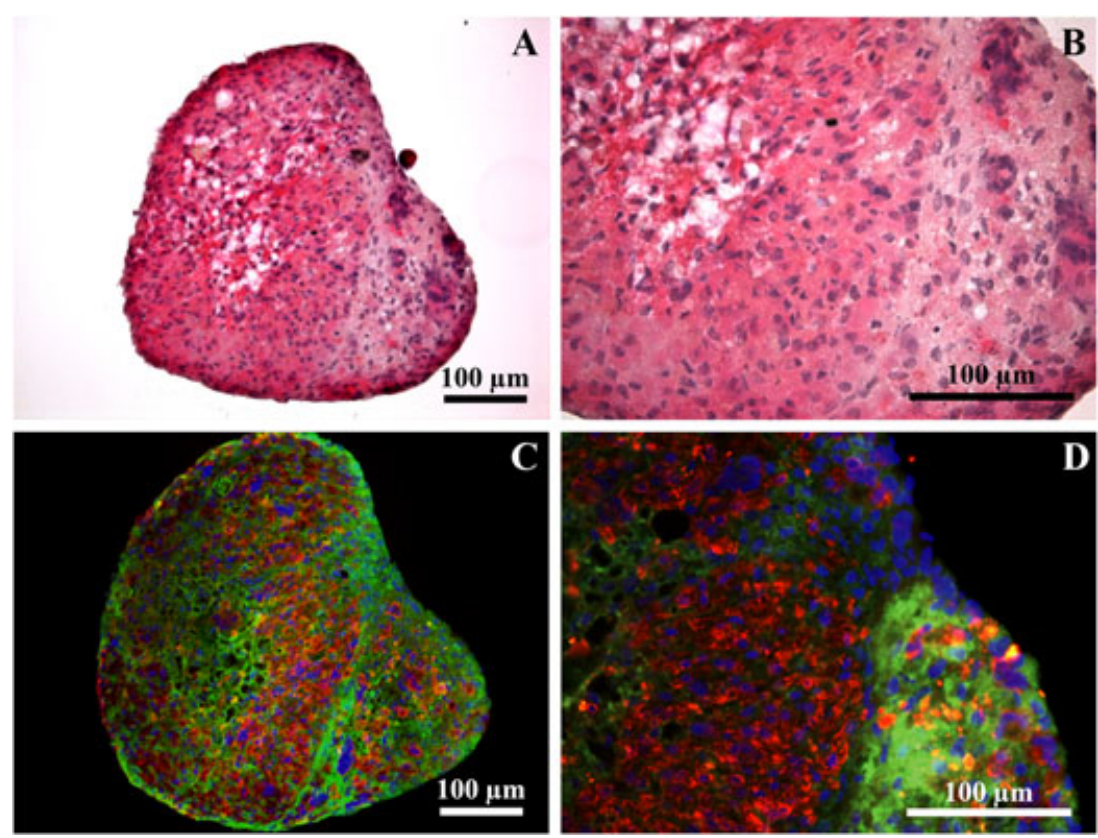

Figure 1. Cross-section of an in vitro muscle construct stained with H\&E (A, B) and for myosin heavy chains (red), collagen I (green) and nuclei (DAPI, blue) (C, D) 


\section{Results}

\subsection{Morphology of muscle constructs}

H\&E staining of the engineered muscle constructs was performed in order to compare general morphology of in vitro constructs (Figure $1 \mathrm{~A}, \mathrm{~B}$ ) with 1 week implanted muscle constructs (Figure 2A, B). Immunostaining was used to visualize sarcomeric myosin heavy chains (red) and collagen type I (green) in the in vitro (Figure 1C, D) compared to implanted (Figure 2C, D) constructs. Cell nuclei were visualized with DAPI (blue). Collagen 1 immunostaining showed a distinct epimysium-like outer layer of collagen formed at the periphery of the explanted constructs (arrows in Figure 2C, D). Additional muscle marker staining for $\alpha$-actinin and nebulin show striations due to developed sarcomeres (Figure 2F, H) and staining for PAX7 showed differentiating multinucleated myosin (Figure 2G). Sarcomeric myosin was present at the higher level in the 1 week implanted compared to in vitro control constructs. Analysis of cross-sections of the constructs using a software package, Image J, showed $20.2 \%$ by area more myofibre staining in the implanted constructs than in in vitro controls $(66.5 \pm 2.1 \%$ of the total area vs $45.3 \pm 4.9 \%$, respectively).
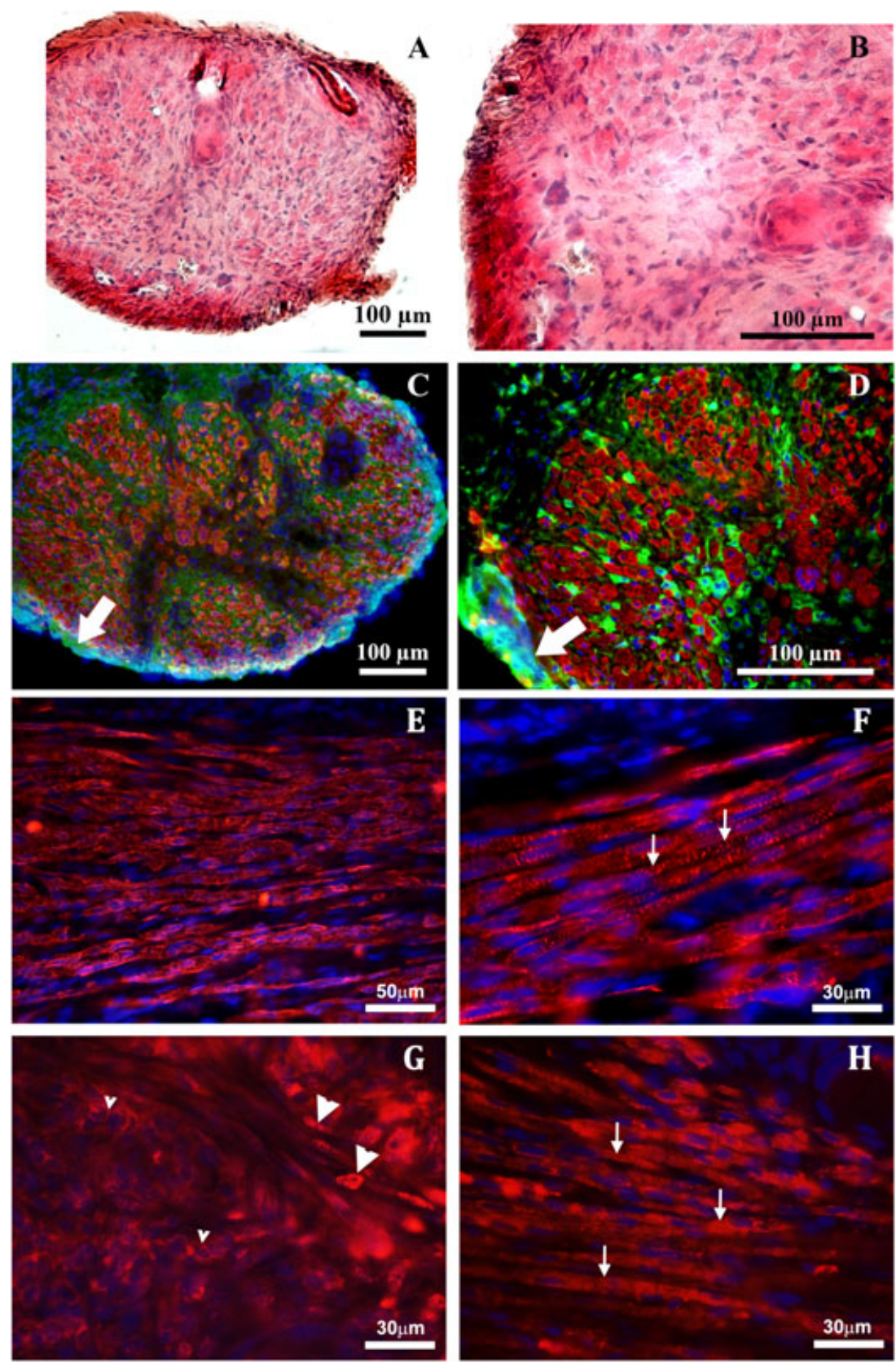

Figure 2. Cross-section of an explanted muscle construct stained with H\&E (A, B) and for myosin heavy chains (red), collagen I (green) and nuclei (DAPI, blue) (C, D). Immunostaining of the longitudinal sections of muscle construct after implantation in vivo with antibodies against MHC (E), $\alpha$-actinin (F), PAX7 (G) and nebulin (H). Nuclei were stained with DAPI (blue in E-H). Small arrows in (F, H) indicate striations due to the presence of the developed sarcomeres. Small arrowheads in (G) indicate perinuclear staining of the non-differentiated myoblasts. Large arrowheads in (G) indicate nuclear staining of the myoblasts in the process of differentiation and fusion 
Longitudinal sections of the implanted muscle constructs stained with antibodies against CD31 indicated that after just 1 week, there was vascularization of the constructs (Figure 3A, B). Immunostaining with antihaemoglobin antibody to demonstrate vessel functionality could not be performed, due to the developing muscle being stained as well, probably due to the cross-reaction with myoglobin (data not shown). However, the CD31 staining provides good evidence of a developing vasculature in 1 week explants. Staining with antibodies against NCAM showed nerve fibres in the sural nerve located in the proximity to the muscle constructs (small arrowheads in Figure 4A, B). There was no NCAM-positive immunostaining in the muscle constructs. Staining with $\alpha$-bungarotoxin for acetylcholine receptors showed intensive staining of the plasma membrane of the developing muscle fibres (arrows in Figure 4A, C, D). In a few areas clustering of the acetylcholine receptors was observed (large arrowheads in Figure 4A, D), suggesting an initiation of neuromuscular junction formation. Immunostaining with antibodies against pan-axonal neurofilament marker showed the presence of nerve fibres in the sural nerve, located in the proximity to the muscle construct (arrow in Figure 4E). We were able to detect nerve fibre sprouting in one section of one muscle construct (arrows in Figure 4F), suggesting outgrowth of axonal processes from the sural nerve into the muscle construct. However, functional testing suggested that 1 week implanted muscle constructs did not have
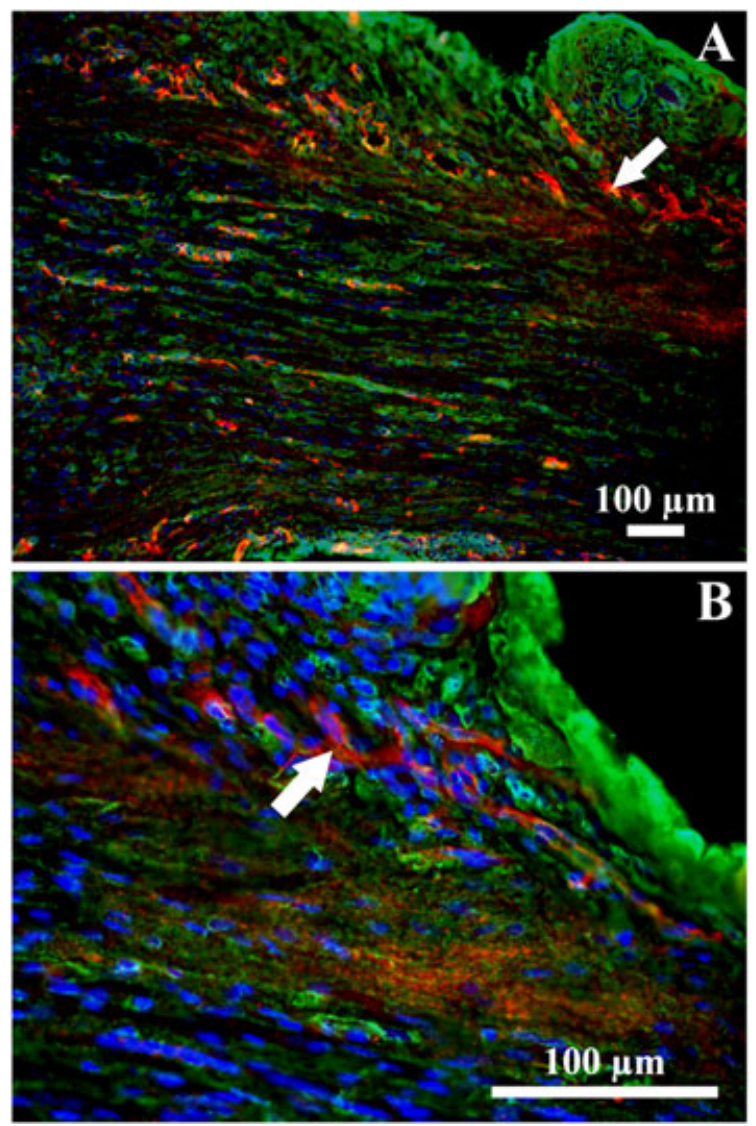

Figure 3. Longitudinal section of an explanted muscle construct stained for blood vessels (CD31, red), connective tissue (WGA, green) and nuclei (DAPI, blue) (A, B) well-formed neuromuscular junctions capable of transmitting action potentials to the muscle. Aortic rings proved an acceptable anchor for the constructs as the aorta-muscle interface stayed intact during all testing (data not shown).

\subsection{Contractile properties}

The muscle constructs exhibited spontaneous baseline activity and could elicit both a twitch and a tetanus response to field stimulation. Following 1 week of implantation, the explanted muscle constructs increased in size, on average from $630 \pm 73 \mu \mathrm{m}$ to $732 \pm 105 \mu \mathrm{m}$, while the in vitro constructs shrank, but not significantly, changing on average from $462 \pm 29 \mu \mathrm{m}$ to $427 \pm 53 \mu \mathrm{m}$ (Figure $5 \mathrm{~A}$ ). The implanted constructs increased maximum isometric force from $192 \mu \mathrm{N}$ to $549 \mu \mathrm{N}$ (245\% increase) compared to in vitro controls that increased from $276 \mu \mathrm{N}$ to 329 $\mu \mathrm{N}$ (25\% increase) over the same time period (Figure 5B, C). The change in isometric force generated between the two groups was significant $(p>0.05)$. Following the field stimulation of the muscle construct, microelectrodes were used to electrically stimulate the neural extensions radiating from the construct. In all nine implanted constructs, there was no response to direct stimulation of the regenerating nerve extension, indicating a lack of functional innervation (data not shown).

\section{Discussion}

The purpose of this research was to investigate the effect of a 1 week in vivo implantation on advancement of phenotype in scaffold-less engineered skeletal muscle constructs. We hypothesized that 1 week of implantation with mechanical stimulation and surgical supplementation of nerve and vasculature would shift the phenotype of the engineered muscle from neonatal toward adult and that muscle constructs would exhibit an increase in force production.

Following 1 week of exposure to the in vivo environment, including mechanical stresses, the muscle constructs increased isometric force production. Previous studies from our laboratory showed that when muscle constructs were exposed to 1 week of innervation with the tendon ends of the construct sutured to the belly of a muscle, it resulted in necrosis of the muscle tissue (data not shown). Although a blood vessel was not routed to the constructs in this pilot study, the constructs were quickly encapsulated in a fibrotic sheath and vascularized. This suggests that the introduction of the blood vessel and/or the mechanical stresses placed on the construct by anchoring it to the tendons of the biceps femoris drove the construct to develop a more adult phenotype. This increase in adult phenotype was concluded from the data measuring a $20.2 \%$ increase in the myosin staining in the muscle tissue. In addition to the increase in myosin staining, we observed that the cross-sections of the implanted construct were larger. Assessment of the 

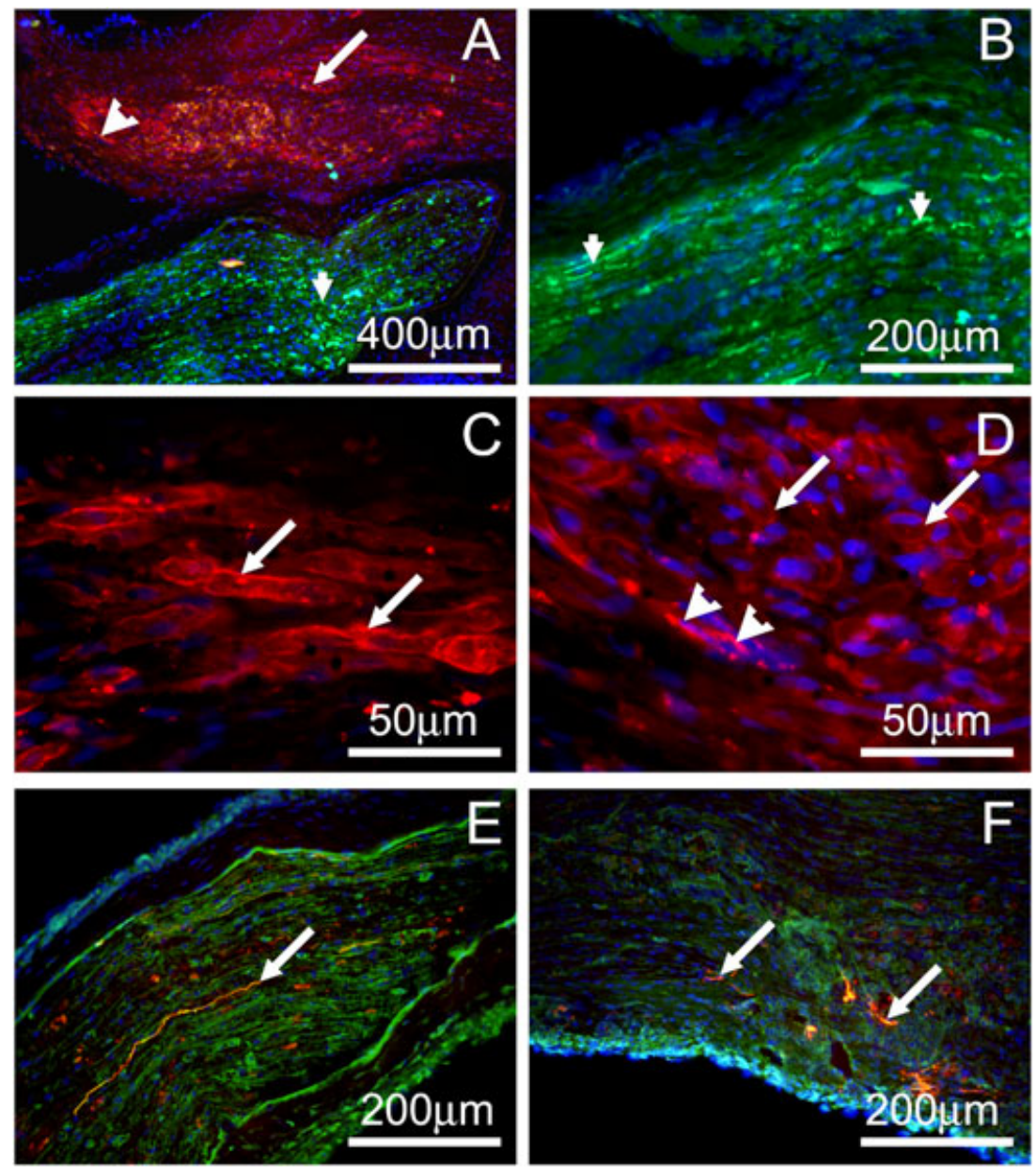

Figure 4. Longitudinal section of an explanted muscle construct stained with antibodies against S100 to visualize nerve fibres (green; A, B), $\alpha$-bungarotoxin to visualize acetylcholine receptors (red; A, C, D) and with antibodies against axonal neurofilament marker (red; E, F). Nuclei were stained with DAPI (blue; A-F). Connective tissue in (E, F) was stained with WGA lectin (green). Small arrowheads in (A, B) indicate nerve fibres in the sural nerve placed in the proximity of the muscle construct. Arrows in $(\mathrm{A}, \mathrm{C})$ indicate developing muscle fibres with $\alpha$-bungarotoxin-stained plasma membrane. Large arrowheads in (A, D) indicate small clusters of acetylcholine receptors at the developing muscle fibres. Arrows in (E, F) indicate nerve fibres in sural nerve and muscle construct, respectively

construct in longitudinal sections indicated an increase in the longitudinal alignment of muscle fibres along the axis of the muscle from end to end.

The beneficial effect of neurotization on muscle tissue development has been studied extensively (Baltich et al., 2010; Das et al., 2010). The appearance of small acetylcholine receptor clusters in our explants in the proximity to the junction of endogenous nerve and engineered muscle tissue suggests that the muscle tissue responds to the regenerating nerve signalling by clustering of acetylcholine receptors. In a similar study performed on scaffolded muscle by Dhawan et al. (2007), scaffolded constructs were implanted with or without nerve transection to promote neurotization of the constructs. In that study, the explanted neurotized constructs generated a force of $649 \mu \mathrm{N}$, while explanted non-neurotized constructs generated only $124 \mu \mathrm{N}$. Thesefindings support our hypothesis that neurotization will play a large role in increasing the maximum isometric force of our constructs, as compared to in vitro controls, given more time in vivo. As stated above, unpublished pilot studies from our laboratory, in which nerve but not vasculature was supplied to the regenerating muscle in vivo, suggest that immediate vascularization may initially play a bigger role in tissue survival than innervation; 2 and 4 week implants have been proposed as a future research goal for our laboratory to improve the level of neurotization and vascularization of the constructs.

Mechanical stimulation has been determined to play an important role in the development of skeletal muscle in vitro. Candiani et al. (2010) showed that 10 days on a bioreactor resulted in an eight-fold increase in myosin accumulation in stretched constructs as compared to static controls. Comparatively, Figures $1 \mathrm{C}$ and $2 \mathrm{C}$ show an increase in staining for myosin heavy chain in implanted constructs as compared to in vitro controls. In support of this data was an increase in force production. Future studies from our laboratory will look at the contribution of mechanical stress on the development of a more advanced phenotype, by placing a subset of engineered muscle constructs on bioreactors prior to implantation. The timing for muscle development must be weighed with the decreased diffusion of substrate into larger more developed muscle constructs in vitro and increased risk 

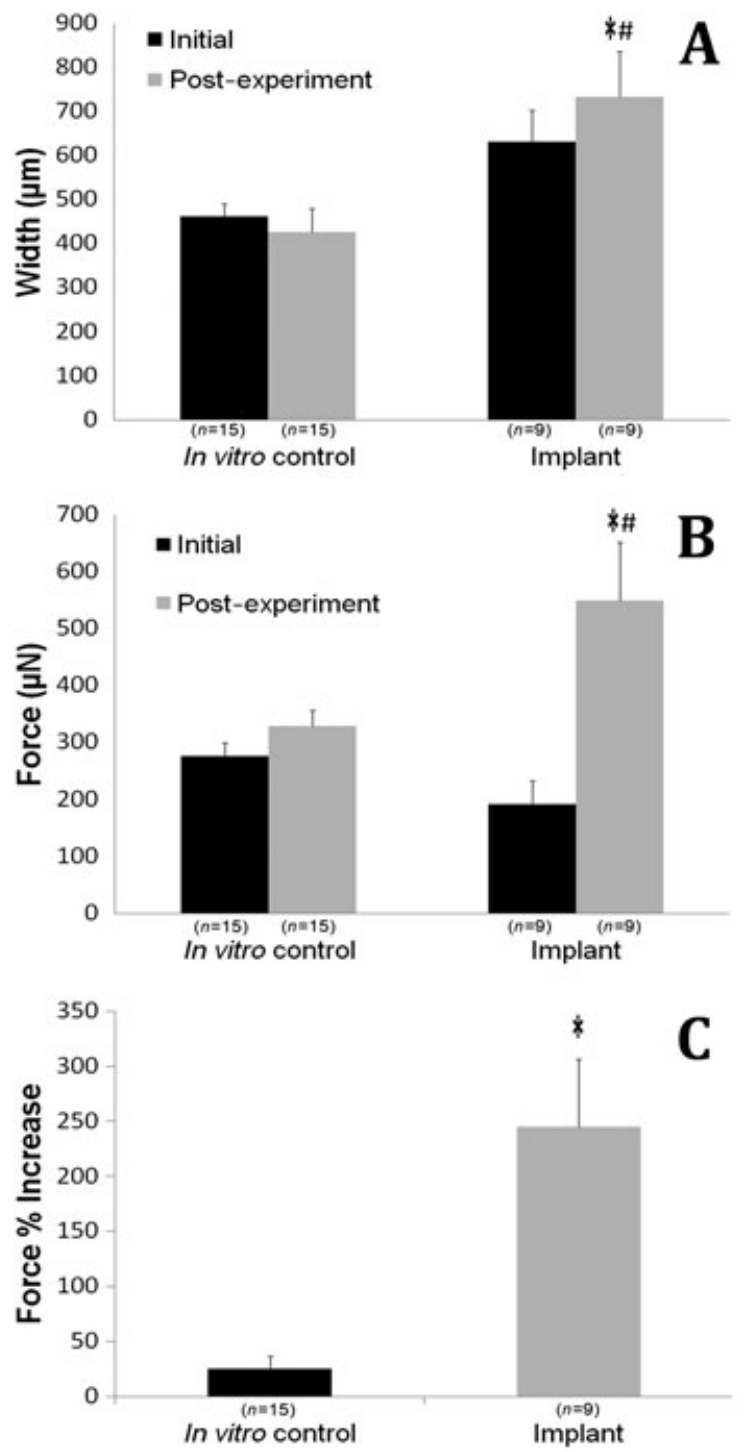

Figure 5. Contractile properties measured prior to (day 0, labelled 'Initial') and 7 days after implantation (day 7, labelled 'Post-experiment') of the muscle constructs; (A) construct width; (B) maximum isometric force; (C) maximum specific force. Values are mean \pm SEM; a oneway ANOVA was performed on all groups to detect significant differences between the in vitro controls vs the implants and the initial (day 0 ) force production vs the day 7 force production. *Significant difference $(p>0.05)$ in implants vs controls at the same experimental time point; ${ }^{\#}$ significant difference $(p>0.05)$ in implants, day 0 vs day 7 experiment

of cell death in the central area of the constructs, due to the lack of nutrients and oxygen.

In conclusion, 1 week of an in vivo environment resulted in constructs with multinucleated muscle fibres and $\alpha$-actinin-stained sarcomeres, as well as greater force-producing capabilities. This advancement of muscle phenotype seen after implantation brings us closer to our goal of using engineered muscle constructs for tissue repair. Future studies in our laboratory will continue to examine the development of the neural and vascular interfaces with our engineered muscle constructs and the viability of replacing whole muscles with engineered tissue.

\section{Acknowledgements}

This research was supported by NIH, NIAMS and NIBIB (Grant No. R01 AR054778-02). We acknowledge Rose Lee and Aaron Adams for technical assistance.

\section{References}

Baltich J, Hatch-Vallier L, Adams AM, et al. 2010; Development of a scaffoldless three-dimensional engineered nerve using a nerve-fibroblast co-culture. In vitro Cell Dev Biol Anim 46: 438-444.
Beier JP, Stern-Straeter J, Foerster VT, et al. 2006; Tissue engineering of injectable muscle: three-dimensional myoblast-fibrin injection in the syngeneic rat animal model. Plast Reconstr Surg 118: 1113-1124.
Candiani G, Riboldi SA, Sadr N, et al. 2010; Cyclic mechanical stimulation favors myosin heavy chain accumulation in engineered skeletal muscle constructs. $J$ Appl Biomat Biomech 8: 68-75. 
Das M, Rumsey JW, Bhargava N, et al. 2010; A defined long-term in vitro tissue engineered model of neuromuscular junctions. Biomaterials 31: 4880-4888.

Dennis RG, Kosnik PE. 2000; Excitability and isometric contractile properties of mammalian skeletal muscle constructs engineered in vitro. In Vitro Cell Dev Biol 36: 327-335.

Dennis RG, Kosnik PE II, Gilbert ME, et al. 2001; Excitability and contractility of skeletal muscle engineered from primary cultures and cell lines. Am J Physiol Cell Physiol 280: 288-295.

Dhawan V, Lytle IF, Dow DE, et al. 2007; Neurotization improves contractile forces of tissue-engineered skeletal muscle. Tissue Eng 13: 2813-2821.

Donnelly K, Khodabukus A, Philp A, et al. 2010; A novel bioreactor for stimulating skeletal muscle in vitro. Tissue Eng C Methods 16: 711-718.

Gonen-Wadmany M, Gepstein L, Seliktar D. 2004; Controlling the cellular organization of tissue-engineered cardiac constructs. Ann NY Acad Sci 1015: 299-311.
Haase SC, Rovak JM, Dennis RG, et al. 2003; Recovery of muscle contractile function following nerve gap repair with chemically acellularized peripheral nerve grafts. $J$ Reconstr Microsurg 19: 241-248.

Huang NF, Lee RJ, Li S. 2010; Engineering of aligned skeletal muscle by micropatterning. Am J Transl Res 2: 43-55.

Irintchev A, Rosenblatt JD, Cullen MJ, et al. 1998; Ectopic skeletal muscles derived from myoblasts implanted under the skin. J Cell Sci 111: 3287-3297.

Khodabukus A, Paxton JZ, Donnelly K, et al. 2007; Engineered muscle: a tool for studying muscle physiology and function. Exerc Sport Sci Rev 35: 186-191.

Kosnik PE, Faulkner JA, Dennis RG. 2001; Functional development of engineered skeletal muscle from adult and neonatal rats. Tissue Eng 7: 573-584.

Kostrominova TY. 2011; Application of WGA lectin staining for visualization of the connective tissue in skeletal muscle, bone, and ligament/tendon studies. Microsc Res Tech 74: $18-22$.
Larkin LM, Calve S, Kostrominova TY, et al. 2006; Structure and functional evaluation of tendon-skeletal muscle constructs engineered in vitro. Tissue Eng 12: 3149-3158.

Larkin LM, Van der Meulen JH, Dennis RG, et al. 2005; Functional evaluation of nerve-skeletal muscle constructs engineered in vitro. In vitro Cell Dev Biol 42: 75-82.

Luna LG. 1968; Manual of Histologic Staining Methods of the Armed Forces Institute of Pathology. McGraw-Hill: New York: 94-95.

National Research Council 1996; Guide for the care and use of laboratory animals. The National Academics Press: Washington, DC.

Powell CA, Smiley BL, Mills J, et al. 2002; Mechanical stimulation improves tissueengineered human skeletal muscle. Am J Physiol Cell Physiol 283: C1557-1565.

Saxena AK, Willital GH, Vacanti JP. 2001; Vascularized three-dimensional skeletal muscle tissue-engineering. Biomed Mater Eng 11: 275-281. 